\title{
Towards a Stray Light Analysis of the ELT
}

\author{
R. Holzlöhner \\ European Southern Observatory (ESO), Karl-Schwarzschild-Str. 2, 85748 Garching, Germany
}

\begin{abstract}
Stray light in telescopes limits the achievable signal-to-noise ratio which is particularly relevant for high-contrast observations. This work analyzes stray light from the Moon and from stars in the field of view that scatters on contaminated telescope mirrors in the ELT.
\end{abstract}

Keywords: ELT, stray light, Mie scattering, dust

\section{OVERVIEW}

Stray light is by definition any unwanted light in an optical system. Stray light in the E-ELT relevant to science can be classified into different categories, as attempted in Fig. 1. The categories with light blue background shading are covered in this work.

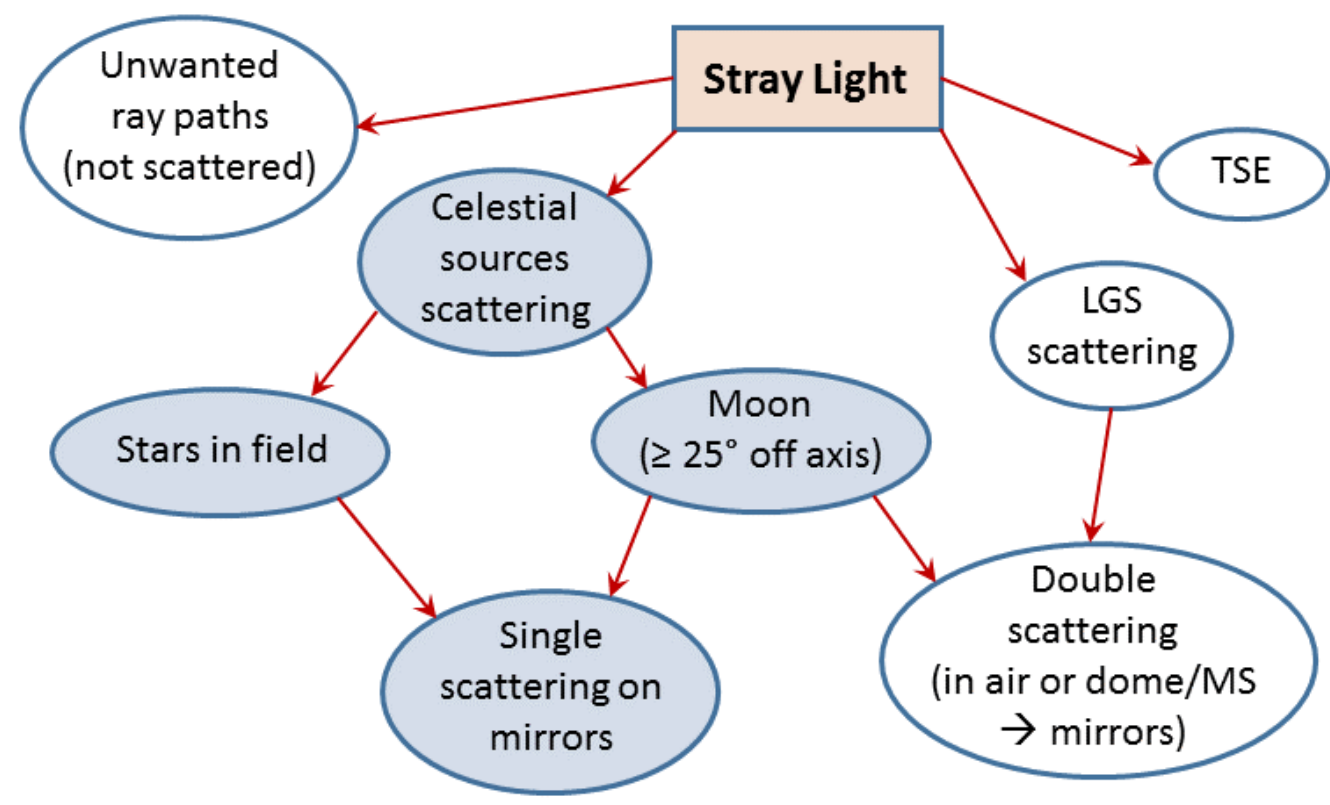

Fig.1: Classification of stray light relevant to E-ELT science. Only classes with shaded background are covered in this work. TSE $=$ Thermal Self-Emission, LGS $=$ Laser Guide Star, MS = (Telescope) Main Structure

\section{INTRODUCTION}

Assuming that the E-ELT instruments all use accurate field and pupil (cold) stops including the spider vanes, the dome is kept free of light sources during observation, and setting aside thermal infrared radiation for the moment, stray light must 
scatter off one of the telescope mirrors. The light either comes directly from a star in the field of view, or else from the Moon. There may be some residual light scattered through the M1 inter-segment gaps and scattering near the prime focus must be studied, but broadly speaking, we can restrict ourselves in this analysis to single scattering events off a mirror (M1-M6N).

The ELT requirement R-L1S-421 specifies that full science operation must be available down to a Moon distance of $25^{\circ}$ off target. The associated off-specular (large angle) scattering is driven mostly by small structures such as dust particles of a few wavelengths diameter and below and surface roughness features of similar size [1]. The off-specular scattering coefficients are very small, but, on the other hand, the Full Moon is an extremely bright object with $m_{V}=-12.5[2,6]$.

Conversely, near-specular scattering, such as within the science field ( 2.5 arcmin radius), is dominated by large structures such as dust particles above $100 \mu \mathrm{m}$ and visible scratches in mirrors, but also mirror edges in the science light path such as at the M1 bevels or the side faces of the segments. Since the scattered light irradiance is a steep function of scatter angle relative to the specular reflection direction, stars far outside the FoV and multiple starlight scattering can be neglected. Understanding this type of scattering is critical for high contrast imaging/spectroscopy, typically done with coronagraphs.

\subsection{Scattering in an Optical System}

Light can scatter off any of the six mirrors M1-M6N and some of the rays will always end up in the focal plane. We will simulate the irradiance of scattered light onto a "virtual pixel" target of size 1 " $\times 1$ " $=(3.31 \mathrm{~mm})^{2}$ centered on the optical axis. The power received on the pixel is given by [10]

$$
P_{\text {pix }}=I_{\text {source }} \cos \theta_{i} \operatorname{BSDF}\left(\theta_{i}, \theta_{s}, \phi\right) A_{\text {refl }} F \Omega_{M, \text { pix }} \cos \theta_{s},
$$

where

$I_{\text {source }}$ is the source irradiance on the ground at normal incidence (unit $\mathrm{W} / \mathrm{m}^{2}$ ),

$\theta_{i}$ is the angle between the source ray and the mirror normal,

$\theta_{s}$ is the angle between the scattered ray and the mirror normal,

$\phi$ is the azimuth between the source and scattered rays projected onto the mirror,

BSDF denotes the Bidirectional Scatter Distribution Function,

$A_{\text {refl }}$ is the reflective area of the mirror,

$F$ is the fraction of $A_{\text {refl }}$ irradiated by the scatter source, and

$\Omega_{M, p i x}$ is the solid angle under which the target is seen from just above the mirror.

The BSDF (unit sr ${ }^{-1}$ ) yields the power fraction of an outgoing ray for a given surface roughness/contamination, geometry $\left(\theta_{i}, \theta_{s}, \phi\right)$ and optical wavelength $\lambda$; see also Subsection 1.4. Equation (1) contains the entire reflective mirror area $A_{\text {refl }}$, hence all rays that can reach the virtual pixel contribute to $P_{\text {pix. }}$. If, instead, one considers a pupil stop in front of the science focal plane, then the scattered rays off a certain mirror have to originate from the footprint on the scattering mirror, pertaining to the field point of the virtual pixel. The footprint is a demagnified copy of the annular pupil whose area $A_{\text {foot }}$ equals $A_{\text {refl }}$ in the pupil (M1), but becomes smaller as one approaches the focal plane (M2-M6N).

Table 1 shows an overview of scatter quantities, where $\alpha$ is the angle between the mirror normal and the optical axis. 
Table 1: Quantifying scattered irradiance originating from M1-M6N

\begin{tabular}{|c|c|c|c|c|l|c|c|}
\hline Mirror & $\begin{array}{c}\text { Angle off } \\
\text { axis } \alpha\left[^{\circ}\right]\end{array}$ & $\begin{array}{l}\Omega_{M, \text { pix }} \\
{\left[\mathrm{as}^{2}\right]}\end{array}$ & $\cos \left(\theta_{\mathrm{s}}\right)$ & $\begin{array}{l}A_{\text {refl }} \\
{\left[\mathrm{m}^{2}\right]}\end{array}$ & $\begin{array}{l}\text { W/o pupil stop } \\
A_{\text {refl }} \Omega_{M, \text { pix }} \cos \left(\theta_{\mathrm{s}}\right) \\
{\left[\mathrm{as}^{2} \mathrm{~m}^{2}\right]}\end{array}$ & $\begin{array}{l}A_{\text {foot }} \\
{\left[\mathrm{m}^{2}\right]}\end{array}$ & $\begin{array}{l}\text { With pupil stop } \\
A_{\text {foot }} \Omega_{M, \text { pix }} \cos \left(\theta_{\mathrm{s}}\right) \\
{\left[\mathrm{as}^{2} \mathrm{~m}^{2}\right]}\end{array}$ \\
\hline M1 & 0 & 1 & 0.98 & 970.97 & 949 & 970.97 & 949 \\
\hline M2 & 180 & 101 & 0.98 & 12.35 & 1214 & 10.44 & 1026 \\
\hline M3 & 0 & 166 & 1. & 11.25 & 1864 & 5.84 & 967 \\
\hline M4 & 172 & 294 & 0.99 & 4.2 & 1228 & 3.33 & 973 \\
\hline M5 & 53 & 510 & 0.78 & 4.85 & 1941 & 2.33 & 931 \\
\hline M6N & 90 & 48405 & 0.71 & 1.19 & 40621 & 0.04 & 1232 \\
\hline
\end{tabular}

It is obvious from Column 6 that the product $A_{\text {refl }} \Omega_{M \text {,pix }} \cos \theta_{\mathrm{s}}$ grows with the mirror number, while $G_{\text {pix }}=A_{\text {foot }} \Omega_{M \text {,pix }}$ $\cos \theta_{\mathrm{s}}$ (Column 8 ) stays roughly constant. This circumstance is a consequence of Lagrange invariance as $G_{\text {pix }}$ can be interpreted as the étendue of the virtual pixel, which is a conserved quantity across all mirrors. The conclusions from this subsection are thus

a) In the absence of a pupil stop, the scattering impact grows steeply with the mirror number (Column $6 ;$ e.g. 43 times larger for M6N than for M1 !)

b) When using a pupil stop and under identical contamination and illumination conditions, the scattering impact from all mirrors is the same

c) The angles $\theta_{i}$ between the source and the mirror normal depend on the mirror orientation. M1, M3, M5 and M6N can be illuminated directly by the Moon.

\subsection{Moonlight}

The Lunar radiation spectrum of the Moon can be rather accurately modeled as the sum of the reflected solar spectrum (reflection best fit at $5050 \mathrm{~K}$ blackbody radiation) and thermal

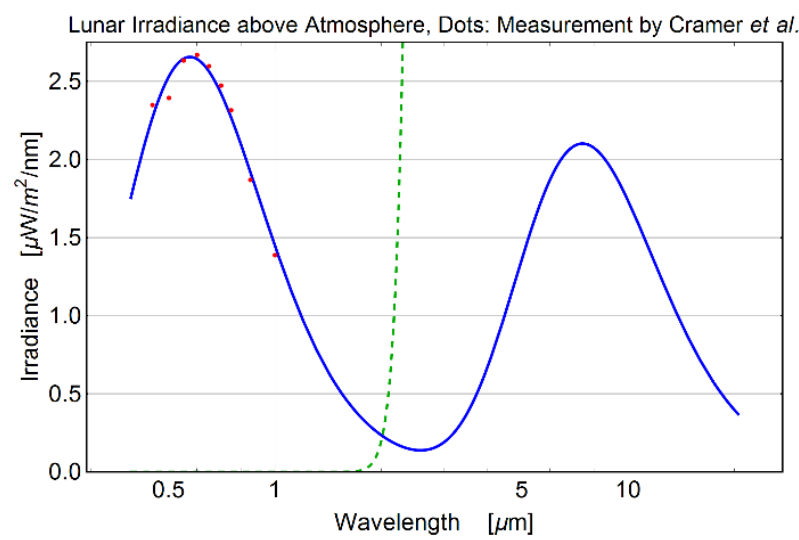

\begin{tabular}{|c|c|c|c|c|}
\hline Band & $\begin{array}{l}\lambda_{\text {cen }} \\
{[\mathrm{nm}]}\end{array}$ & $\begin{array}{l}\Delta \lambda \\
{[\mathrm{nm}]}\end{array}$ & $\begin{array}{l}\text { Photon flux on } \\
\text { ground }(X=1) \\
{\left[10^{15} \mathrm{ph} / \mathrm{s}^{2} \mathrm{~m}^{2}\right]}\end{array}$ & $\begin{array}{l}\text { magnitude } \\
\text { (in band) }\end{array}$ \\
\hline $\mathrm{U}$ & 365 & 66 & 0.11 & -11.4 \\
\hline $\mathrm{B}$ & 445 & 94 & 0.39 & -11.3 \\
\hline $\mathrm{V}$ & 551 & 88 & 0.6 & -12.2 \\
\hline $\mathrm{R}$ & 658 & 138 & 1.11 & -12.7 \\
\hline $\mathrm{I}$ & 806 & 149 & 1.23 & -13.1 \\
\hline $\mathrm{Y}$ & 1020 & 120 & 0.85 & -13.4 \\
\hline $\mathrm{J}$ & 1220 & 213 & 1.22 & -13.7 \\
\hline $\mathrm{H}$ & 1630 & 307 & 1.09 & -13.9 \\
\hline $\mathrm{K}$ & 2190 & 390 & 0.79 & -14.1 \\
\hline $\mathrm{L}$ & 3450 & 472 & 2.94 & \\
\hline $\mathrm{M}$ & 4750 & 460 & 13.19 & \\
\hline $\mathrm{N}$ & 10500 & 2500 & 213.84 & \\
\hline $\mathrm{Q}$ & 21000 & 5800 & 214.7 & \\
\hline
\end{tabular}

Fig. 2: Full Moon irradiance above the atmosphere vs. wavelength. Left: Log-linear plot, Right: Table of photon flux vs. band ( $\lambda$ : band center wavelength, $\Delta \lambda$ : FWHM wavelength range) 
Figure 2 also includes a table of the associated photon flux on the ground with the Moon at zenith (airmass $X=1$ ) across some astronomical bands. The last column shows the corresponding star magnitudes relative to $m=0$ in each respective band. It is interesting to note that while the irradiance e.g. in K-band $(2.2 \mu \mathrm{m})$ is only about one tenth of that in the Vband $(0.55 \mu \mathrm{m})$, the photon flux in $\mathrm{K}$ is even larger than in $\mathrm{V}$. This situation is a consequence of bands widening in the infrared (390 nm vs. $88 \mathrm{~nm}$ in the example) and the photon energy scaling like $1 / \lambda$.

The dependence of Lunar irradiance on Earth versus Lunar phase angle is shown in Fig. 3 in the Appendix.

\subsection{Dust on Mirrors}

Typical mirrors of ground-based telescopes are rather dirty compared to those in most other optical systems. Contaminants mainly consist of small mineral particles like silica, but also include thin grease/oil films [11].

Experience on the VLTs shows that the average throughput M1-M3 is about $65 \%$ in the visible. Assuming a reflectivity of a freshly oxidized aluminium coating of $90.5 \%$ at $589 \mathrm{~nm}$, reflectivity losses of e.g. $7 \%$ on $\mathrm{M} 1,1 \%$ on $\mathrm{M} 2$ and $3.5 \%$ on M3 would explain this figure. By comparison, the combined area of all M1 inter-segment gaps and bevels totals only about $1 \%$ of the pupil area $\left(9.1 \mathrm{~m}^{2} / 970 \mathrm{~m}^{2}=0.93 \%\right)$, showing that the net telescope reflectivity loss, compared to the state of all monolithic and freshly coated mirrors, is mainly driven by dust. The effects of residual mirror polishing roughness and M1 segmentation are secondary.

The Optics Group in Paranal has run a multi-year study of mirror witness samples, both inside the VLT domes in cages and outside in a well ventilated hut near the DIMM Tower [3]. The result is that the specular reflectivity loss in the visible in percent per 100 days can be approximated by the fit function

$$
\Delta R=0.9 \max \{\cos (\alpha), 0\}+0.1
$$

where $\alpha$ is the angle between the mirror normal and the telescope optical axis (i.e., $\alpha=0$ for upward facing mirrors, $\alpha=45^{\circ}$ for M3 in the VLT etc.) and $\max \{$,$\} yields the maximum of two arguments. The three mirrors in the VLT thus$ accumulate dust at approximate rates of M1: 1\%, M2: $0.1 \%$ and M3: $0.73 \%$ per year, ignoring that M3 is retracted when observing at Cassegrain focus, protected from dust. The reflectivity loss rate of $1 \%$ per year in the visible due to dust was also confirmed by a multi-year mirror study on silver coated mirrors at Gemini South [11].

Requirement R-L1S-418 of the E-ELT Level-1 Requirements is based on the cleanliness class CL630 defined in the standard [4]. This standard describes a lognormal dust particle size distribution that was found in 1962 to be appropriate for recently cleaned optics in US military cleanrooms. The minimum assumed particle diameter is $1 \mu \mathrm{m}$ and towards the long tail there is a single particle of $630 \mu \mathrm{m}$ diameter or more per square foot on average.

The fractional geometrical dust coverage of CL630 is 1.0\%. However, the associated loss of specular reflection in the far field is actually $2 \%$, not $1 \%$, caused by the combination of backward reflection, absorption and diffraction [1, Section 4.4.3]. In the visible, these $2 \%$ scatter diffusely. On the other hand, in the (far) infrared, where many particles become smaller than the wavelength, absorption becomes relevant and the total absorbed power is comparable to the scattered power. This last point becomes relevant for TSE calculations.

Geometric area coverage is difficult to measure directly (requires classifying/counting particles under the microscope), but needed for the numerical simulation of Mie scattering. Specular reflectivity in the visible, on the other hand, can be measured in situ with rather affordable commercial reflectometers.

The EELT recoating baseline schedule at the time of writing foresees that each M1 segment is recoated every 18 months and M3 shall be washed or recoated every two years.

We can draw the following preliminary conclusions from this section:

a) The standard IEST1246D [4] is not well suited for ground-based telescope mirrors, which are not in a cleanroom and most of the time far from the "recently cleaned" state.

b) On a single upward facing mirror, $1 \%$ specular reflectivity loss $=0.5 \%$ area coverage is reached in the Atacama after about 100 days (hence CL630 after 200 days). 
c) Exposing the E-ELT mirrors M1-M6N with the orientation angles $\left\{0^{\circ}, 180^{\circ}, 0^{\circ}, 172^{\circ}, 53^{\circ}, 90^{\circ}\right\}$ for 100 days to dust, one suffers $3 \%$ total specular reflectivity loss (equivalent to " 1.5 times CL630")

d) With the baseline of recoating M1 segments every 18 months, the time averaged segment dust exposure is 548/2 $=274$ days $=9$ months $\rightarrow 2.74 \%$ reflectivity loss. Assuming that the other mirrors are cleaned/recoated every 24 months $(\rightarrow 365$ days avg. exposure), we experience a time average of $\mathbf{1 0 \%}$ telescope specular reflectivity loss, and thus emissivity increase, due to dust (equivalent to "5 times CL630")

\section{4 $\quad$ BSDFs}

In this analysis, we consider Mie scattering according to the standard IEST1246D [4] with the default slope of 0.926. In the future, more relevant particle distribution functions or, optimally, measured scattering functions should be used. As stated before, mirror-polishing roughness also generates scatter, but can be neglected in a first analysis because dust dominates, except directly after recoating.

In order to magnify the near-specular scatter behavior (a few arcminutes off the specular ray), but also to generalize to out-of-plane scattering (hence $\phi \neq 0$ ), BSDFs are usually plotted against the quantity $\left|B-B_{0}\right|=\left|\sin \theta_{s}-\sin \theta_{i}\right|$ which is limited to the range $(0,2)$. Since the absolute value $\left|B-B_{0}\right|$ cannot distinguish between forward $\left(B>B_{0}\right)$ and backward $\left(B<B_{0}\right)$ scatter directions, the curves in the corresponding plots consist of two branches. Both scatter branches eventually peak upward, corresponding to the peaks seen at the left and right edges of the plots against linear scatter angle (Scatter Angle $=\theta_{s}= \pm 90^{\circ}$ ). At large angle scattering (i.e., far from specular), forward Mie scattering dominates over backward scattering. If the scattering particle sits on a mirror, some forward scattered rays reflect off the mirror, but backward scattered light does not.

Two Mie scatter BSDFs computed in the FRED simulation software are shown in Fig. 4 of the Appendix. The curves peak at the specular reflection direction and subtend a vertical range of about five decades and more at oblique incidence angles (note the log scale on the vertical axes).

The integration of the BSDF over the hemisphere yields the Total Integrated Scatter (TIS) [10]. The approximate relationship TIS $=2 \times \mathrm{PAC} / \cos \left(\theta_{i}\right)$ holds in the visible in percent, where PAC (Percent Area Coverage) is the geometrical area coverage in percent, hence e.g. PAC $=1.0$ for CL630.

Besides scattering, dust also absorbs light, modeled by the imaginary part of the refractive index of the particles. The total absorption can be quantified as TIA (Total Integrated Absorption). According to Mie scatter theory, the sum of TIS and TIA equals twice PAC, independent of wavelength. It should be mentioned that this theoretical result seems to disagree with measurements that usually report increasing specular reflectivity loss with decreasing wavelengths [12]. The topic is under investigation.

The BSDF curves generally become flatter towards increasing wavelength with diminished peaks for near-specular scattering, and grow at large-angle (off-specular) scattering, as evident in the four plots shown in Fig. 4, valid for $551 \mathrm{~nm}$ (center of V-band, upper two plots) vs. $2.2 \mu \mathrm{m}$ (center of K-band, lower two plots). All BSDFs are for CL630 (1\% geometrical area coverage $\rightarrow 2 \%$ specular reflectivity loss). The curves scale linearly with the PAC.

One can draw the following conclusions from this subsection:

a) Mie scattering on dusty mirrors is strongly angle dependent, with near-specular scatter larger than off-specular by $3-4.5$ orders of magnitude within $\mathrm{V}-\mathrm{K}$,

b) Near-specular Mie scattering scales roughly like $\lambda^{-2}$, while off-specular is only weakly wavelength dependent within $\mathrm{V}-\mathrm{K}$,

c) The near-specular BSDF values should be considered lower limits since this scatter regime is dominated by a small number of large irregularities in the telescope beam (large dust particles, mirror scratches/chips, segment bevels, spider vane edges etc.). 


\begin{tabular}{|c|c|c|c|}
\hline Band & $\begin{array}{c}\text { near-specular } \\
{\left[\mathrm{sr}^{-1}\right]}\end{array}$ & $\begin{array}{c}\text { off-specular } \\
\left(\theta_{i}=30^{\circ}, \theta_{s}=0^{\circ}\right) \\
{\left[10^{-3} \mathrm{sr}^{-1}\right]}\end{array}$ & $\begin{array}{c}\text { off-specular } \\
\left(\theta_{i}=60^{\circ}, \theta_{s}=0^{\circ}\right) \\
{\left[10^{-3} \mathrm{sr}^{-1}\right]}\end{array}$ \\
\hline $\mathrm{V}$ & 80 & 2.76 & 1.28 \\
\hline $\mathrm{R}$ & 52.5 & 2.95 & 1.45 \\
\hline $\mathrm{J}$ & 15.5 & 3.38 & 1.58 \\
\hline $\mathrm{K}$ & 5.0 & 3.05 & 1.39 \\
\hline
\end{tabular}

\section{RESULTS}

We quantify the scattering by calculating the photon flux onto the virtual pixel in the focal plane.

\subsection{Moonlight Scattering}

According to the preceding subsections, we can rewrite Eq. (1) for the number of photons per time from Moonlight scattering onto the virtual pixel $(1 " \times 1 ")$ can be written as a sum over the mirrors $k$

$$
n_{\text {pix }}=\Phi_{\text {Moon }} G_{\text {pix }} \sum_{k} \cos \theta_{i, k} \operatorname{PAC}_{k} F_{k} \operatorname{BSDF}_{\mathrm{CL} 630}\left(\theta_{i, k}, \theta_{s, k}, \phi_{k}\right),
$$

where the Lunar photon flux $\Phi_{\text {Moon }}$ is given in the table in Fig. 2 and $G_{\text {pix }}$ in Subsection 1.1. The Moon is assumed to be at least $25^{\circ}$ off target and can directly hit M1, M3, M5 and, if deployed, M6N. The first three mirrors also accumulate dust the fastest, so we employ Eq. (2) and obtain $\mathrm{PAC}_{1}=\mathrm{PAC}_{3}=2$ for $\mathrm{M} 1$ and $\mathrm{M} 3$ and $\mathrm{PAC}_{5}=2\left(0.9 \cos \left(53^{\circ}\right)+0.1\right)$ $=1.3$ for M5.

M6N is somewhat special since its surface normal lies always in the $\mathrm{x} / \mathrm{y}$ plane, hence it can only receive direct Moonlight for certain azimuthal orientations to the Moon. However, in these cases it scatters a lot of light towards the instruments due to its proximity to the focal plane with a large solid angle of 48,405 $\operatorname{arcsec}^{2}$. Example: Let the Moon be at $60^{\circ}$ zenith angle and at around $45^{\circ}$ azimuth relative to the dome slit, shining towards Nasmyth A. Then $\theta_{i}=30^{\circ}$ and $\mid B$ $-B_{0} \mid=0.866$, corresponding to $60^{\circ}$ off-specular angle (this angle is between two rays without common plane, however). Assuming $\mathrm{PAC}_{\mathrm{M} 6 \mathrm{~N}}=0.2$ (vertical mirror) and using Table 2, we find $n_{\text {pix }}=3.7-7.3 \times 10^{5}$ photons $/ \mathrm{s}$ from M6N only from $\mathrm{V}-\mathrm{K}$ bands in the absence of a pupil stop. This photon flux is about twice the scatter flux from all mirrors combined, but with a pupil stop, raising the concern that stray light may leak through despite the stops.

Table 3 shows $n_{\text {pix }}$ for two cases of Full Moon scattering on E-ELT mirrors for the bands V-K: The first case (columns 4-6) is rather pessimistic and assumes that the Moon is only $25^{\circ}$ off target, and M1, M3, M5 and M6N receive direct and unobscured Moonlight. The second case (columns 7-9) is more optimistic, assuming the Moon is $60^{\circ}$ off target, M3 is obscured $50 \%$ by the ARU Tower structure $\left(F_{3}=0.5\right)$, and M6N does not receive direct Moonlight $\left(F_{6}=0\right)$.

Diffusely scattered light is uniformly distributed across the field and looks like an elevated sky background whose in band magnitudes are printed in columns 4 and 5. In order to generalize the tabulated values to other Moon phases, one can consult Fig. 3 (e.g., Half Moon $\rightarrow$ phase angle $90^{\circ} \rightarrow \approx 2$ V-band magnitudes penalty). 
Table 3: Stray light onto virtual pixel (1"×1") from Moonlight scattering on contaminated E-ELT mirrors

\begin{tabular}{|c|c|c|c|c|c|c|c|c|}
\hline Band & $\begin{array}{l}\lambda_{\text {cen }} \\
{[\mathrm{nm}]}\end{array}$ & $\begin{array}{l}\Delta \lambda \\
{[\mathrm{nm}]}\end{array}$ & $\begin{array}{c}\text { Photons on pixel } \\
\text { (25 off target) } \\
\text { [ph/s] }\end{array}$ & $\begin{array}{l}\text { magnitude } \\
\text { (in band) } \\
{\left[\mathrm{as}^{-2}\right]}\end{array}$ & $\begin{array}{l}\text { scattered } \\
\text { Moonlight / sky } \\
\text { background [\%] }\end{array}$ & $\begin{array}{c}\text { Photons on pixel } \\
\left(60^{\circ} \text { off target) }\right. \\
\text { [ph/s] }\end{array}$ & $\begin{array}{l}\text { magnitude } \\
\text { (in band) } \\
{\left[\mathrm{as}^{-2}\right]}\end{array}$ & $\begin{array}{l}\text { scattered } \\
\text { Moonlight / sky } \\
\text { background [\%] }\end{array}$ \\
\hline $\mathrm{V}$ & 551 & 88 & 184300 & 19.1 & 24 & 53100 & 20.5 & 14 \\
\hline$R$ & 658 & 138 & 337500 & 18.6 & 37 & 97300 & 19.9 & 24 \\
\hline I & 806 & 149 & 369700 & 18.2 & 53 & 106600 & 19.5 & 33 \\
\hline $\mathrm{Y}$ & 1020 & 120 & 254200 & 17.8 & 44 & 73300 & 19.2 & 20 \\
\hline $\mathrm{J}$ & 1220 & 213 & 363500 & 17.6 & 38 & 104800 & 19. & 14 \\
\hline $\mathrm{H}$ & 1630 & 307 & 325300 & 17.4 & 7 & 93800 & 18.8 & 2 \\
\hline $\mathrm{K}$ & 2190 & 390 & 235300 & 17.2 & 16 & 67800 & 18.5 & 5 \\
\hline
\end{tabular}

Columns 6 and 9 show the total scattered Moonlight onto the virtual pixel at $25^{\circ}$ and $60^{\circ}$ off target, divided by the sky background under the respective conditions versus the band.

\subsection{Near-Specular Scattering from Stars in FoV}

The E-ELT science FoV has a diameter of $2.5^{\prime}=0.73 \mathrm{mrad}$. The BSDFs shown in Fig. 4 level off at $\left|B-B_{0}\right|=1.16 \mathrm{mrad}$ $=4$ '. Consequently, when the telescope observes an object of interest near the field center, any star within the technical field scatters with the same attenuation (leakage) factor onto the image of this object. The scatter attenuation factor can be considered a contrast limit, as shown in Table 4 for the bands V-K. In the last column, "delta magnitude" denotes the factor by which the light from a star in center field is attenuated anywhere else in the science field.

The requirements of the planned exoplanet instrument EPICS specify contrasts of better than $10^{-8}$ at 30 mas and $10^{-9}$ beyond 100 mas angular separation within 600-1650 nm [5]. In order to achieve these requirements, one has to reduce the pixel scale, e.g. to 10 mas, which reduces the scattered light power per pixel by $10^{-4}$. If the object PSF is fully contained within a single pixel (the E-ELT diffraction limit at $1 \mu \mathrm{m}$ is 6.8 mas), a raw contrast of a few parts in $10^{-10}$ becomes possible in principle, ignoring the $\mathrm{AO}$ halo and all other error sources. In reality, scattering from contaminated mirrors may become a leading limiting factor for high-contrast ELT observations.

Table 4: Stray light onto virtual pixel (1” $\times 1$ ”) from in-field starlight scattering on dusty E-ELT mirrors

\begin{tabular}{|c|c|c|c|c|}
\hline Band & $\begin{array}{l}\lambda_{\text {cen }} \\
{[\mathrm{nm}]}\end{array}$ & $\begin{array}{l}\Delta \lambda \\
{[\mathrm{nm}]}\end{array}$ & $\begin{array}{c}\text { contrast limit } \\
(1 " \times 1 " \text { pixels }) \\
{\left[\times 10^{-6}\right]}\end{array}$ & $\begin{array}{l}\text { delta } \\
\text { magnitude }\end{array}$ \\
\hline $\mathrm{V}$ & 551 & 88 & 8.9 & 12.6 \\
\hline $\mathrm{R}$ & 658 & 138 & 5.1 & 13.2 \\
\hline $\mathrm{I}$ & 806 & 149 & 2.1 & 14.2 \\
\hline $\mathrm{Y}$ & 1020 & 120 & 1.6 & 14.5 \\
\hline $\mathrm{J}$ & 1220 & 213 & 1.7 & 14.4 \\
\hline $\mathrm{H}$ & 1630 & 307 & 1.6 & 14.5 \\
\hline $\mathrm{K}$ & 2190 & 390 & 0.6 & 15.6 \\
\hline
\end{tabular}




\section{CONCLUSIONS}

This paper contains a preliminary analysis of stray light in the E-ELT, focusing on single scattering of moonlight and starlight on contaminated mirrors in $\mathrm{V}-\mathrm{K}$ bands.

The E-ELT differs from other ESO telescopes in a number of ways that makes it more prone to scattered stray light off its mirrors: It contains 5-6 serial mirrors to the instruments instead of 2-3, of which 3 to 4 can be directly illuminated by the Moon. The "dome F-number" (ratio of dome slit edge height above M1 to dome slit width) is only 1.24, versus e.g. 1.60 in the VLT, hence the ELT mirrors see a larger solid angle of the sky. The ELT does not have a moonshield, as for instance VISTA does. Finally, cleaning the M1 segments, e.g. by $\mathrm{CO}_{2}$ snow, is difficult.

The following key results were found:

1. Accurate pupil stops in the E-ELT instruments are crucial, in particular to block stray light from scattering on the mirrors closest to the focal plane

2. In the presence of accurate stops and baffles, dust on mirrors is a leading cause of stray light in $\mathrm{V}-\mathrm{K}$ bands and contributes identical amounts of power onto the focal plane, irrespective of which mirror (M1-M6N) it sits on

3. Dust fallout on upward facing mirrors in the Atacama reduces the specular reflectivity by about $1 \%$ per 100 days per mirror ("50\% of CL630 contamination")

4. Given the current baseline washing/recoating schedule, one can expect a total time-averaged reflectivity loss in the ELT of about $10 \%$ due to dust

5. Off-specular scattering (e.g. from Moonlight) is almost independent of wavelength, but near-specular scattering (starlight within field) is 3-4.5 orders of magnitude more efficient and scales like $\lambda^{-2}$

6. Scattered Moonlight under adverse conditions (Full Moon $\leq 60^{\circ}$ off target shining fully onto M1, M3, M5 and $\mathrm{M} 6 \mathrm{~N})$ can raise the apparent sky background above $1 \mu \mathrm{m}(\mathrm{Y}-\mathrm{K})$ by up to $44 \%$

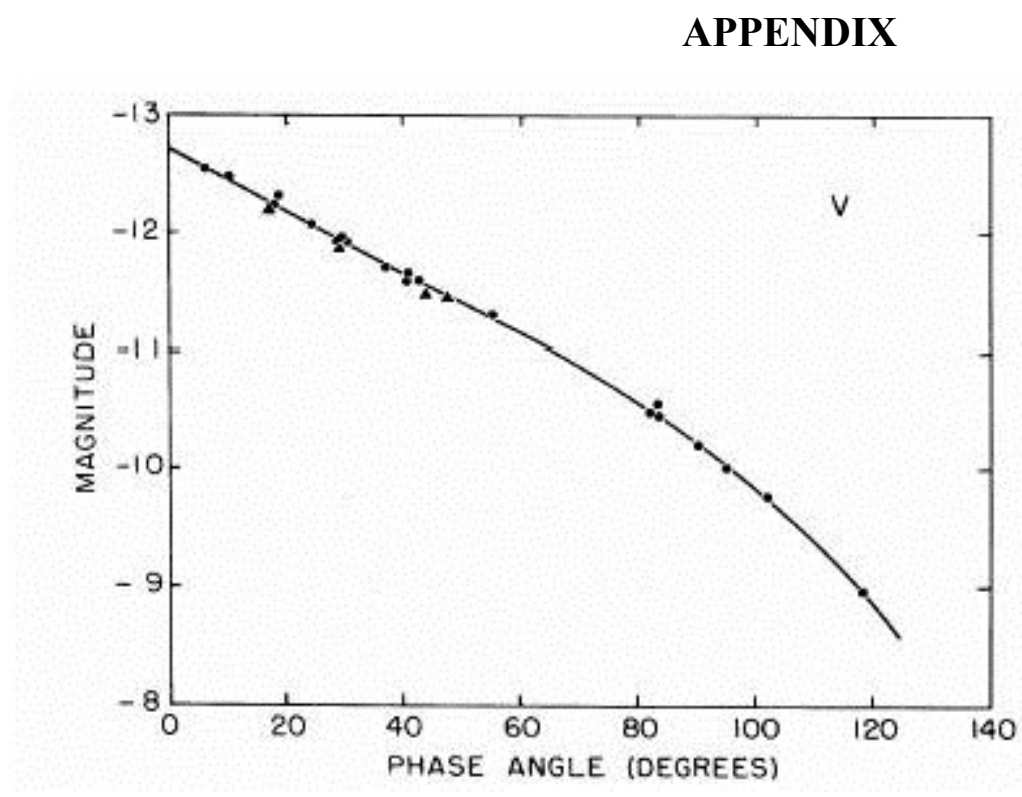

Fig.3: Apparent Lunar V-band magnitude versus phase angle (Source: [6]) 


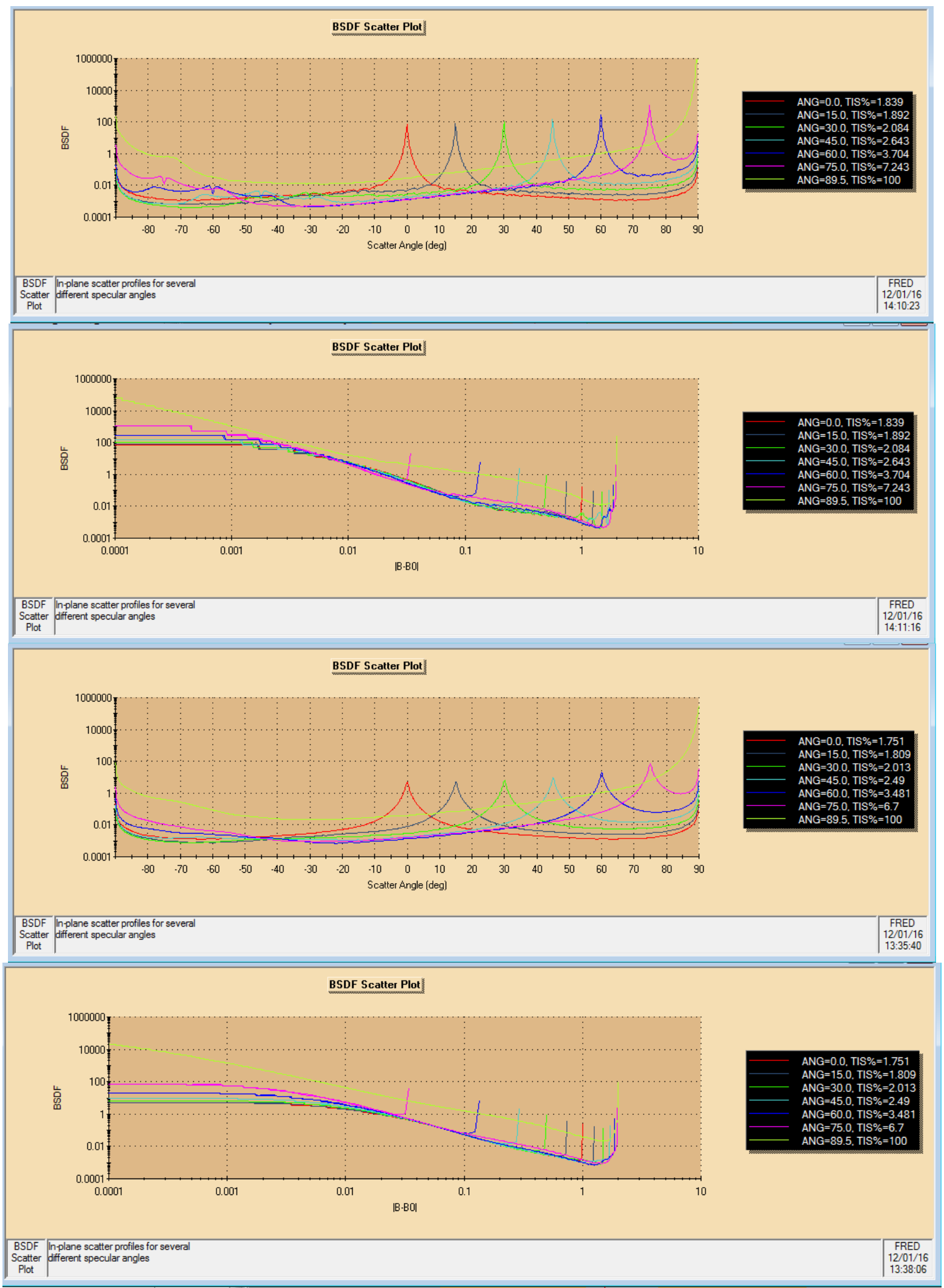

Fig.4: BSDF plots from FRED for $551 \mathrm{~nm}$ (V-band, top 2) and $2.2 \mu \mathrm{m}$ (K-band, bottom 2) vs. linear angle and |B-B0| 


\section{REFERENCES}

[1] C. F. Bohren and D. R. Huffman, Absorption and Scattering of Light by Small Particles, Wiley (1983).

[2] C.E. Cramer, K.R. Lykke, J.T. Woodward, A.W. Smith, "Precise Measurement of Lunar Spectral Irradiance at Visible Wavelengths", J. of Res. NIST 118, p.396-402. PMID 26401440 DOI: 10.6028/jres.118.020 (2013).

[3] S. Guisard, "Study of the effect of dust deposition on Paranal mirrors - Report 2014", Internal ESO draft report (2014).

[4] IEST-STD-CC1246D Product Cleanliness Levels and Contamination Control Program http://electroiq.com/blog/2005/08/iest-std-cc1246d-product-cleanliness-levels-and-contamination-controlprogram/

[5] M. Kasper, J.-L. Beuzit, et al., "EPICS: direct imaging of exoplanets with the E-ELT", Proc. SPIE 7735 (2010).

[6] M. Luciuk, "How Bright is the Moon?", online Tutorial 26, Sperry Observatory http://www.asterism.org/tutorials/tut26-1.htm.

[7] J. A. Shaw, "Modeling infrared lunar radiance", Opt. Eng. 38, p.1763-1764 (1999).

[8] SkyCalc, Online ESO exposure and sky background calculator http://www.eso.org/observing/etc/skycalc/

[9] P.R. Spyak and W.L. Wolfe, "Scatter from particulate-contaminated mirrors. Part 1: theory and experiment for polystyrene spheres and A $=0.6328 \mu \mathrm{m}$ ", Opt. Eng. 31, 1746-1756 (1992).

[10] J.C. Stover, Optical Scattering Measurements and Analysis, SPIE Press, 3rd Ed, ISBN: 9781628418408 (2012).

[11]T. Vucina; M. Boccas; C. Araya; C. Ahhee, "Gemini's protected silver coatings: first two years in operation", Proc. SPIE 6273, 62730W (2006); doi: 10.1117/12.670866.

[12]T. Schneider, T. Vucina, C. Araya, et al., "The Gemini Observatory protected silver coating: ten years in operation", Proc. SPIE 9906 (2016) 